\title{
Research with Stored Ions Produced Using Synchrotron Radiation
}

D. A. Church and S. D. Kravis

Physics Department

Texas A\&M University

JAN2 251988

College Station, Texas r7849, USA

\author{
M. Meron, B. M. Johnson, and K. W. Jones \\ Brookhaven National Laboratoty \\ Upton, New York 11979, USA
}

\author{
I. A. Sellin, C. S. O, J. C. Levin; and R. T. Short \\ Dhysics Department \\ University of Tennessee \\ Knoxville, Tennessee 97999, USA \\ and Physics Division, Oak Ridge National Laboratory \\ Oak Ridge, Tennessee s7891, USA
}

\begin{abstract}
A distribution of argon ion charge states has been produced by inner shell photoionization of argon atoms using $x$-ray synchrotron radiation. These ions were stored in a Penning ion trap at moderate to very low well depths, and analog-detected yielding narrow charge-to-mass spectrum linewidths. Estimates of ion densities indicated that ion-ion collisional energy transfer should be rapid, leading to thermalization. Measurements using variants of this novel stored, multi-charged jon gas are considered.
\end{abstract}




\section{Introduction}

Considerable interest currently exists in both collision and spectroscopic studies of highly-charged ions at Jow energies, where Doppler broadening and shifts are small, and where quantum mechanical charge transfer cross section calculations are most accurate. Studies of ion production using synchrotron radiation, and the storage of the resulting charges as a multi-charged ion gas near room temperature are discussed here; these results are expected to lead to interesting developments in several areas.

A synchrotron $x$-ray source provides both the photon energy and tlux to efficiently eject inner shell electrons from atoms, while transfering minimal recoil energy to the nucleus. K-shell photoionization of argon atoms, followed by a vacancy cascade, results in ions with a distribution of charge states (1) peaked at $A r^{4+}$, with $A r^{5+}$ and $A r^{3}+$ the next most probable charge states. Charge-to-mass spectrum line widths observed in time-offlight measurements (2) demonstrate that the ions are produced with a distribution of initial energies close to room temperature. Confinement of these ions in a manner which preserves the low energies and provides means for subsequently increasing their charge states (3) has now been demonstrated.

\section{Technique and Measurements}

A Penning ion trap was installed on the X-26C beam line of the Atomic Physics Facility (4) at the National Synchrotron Light Source at Brookhaven National Laboratory. The trap was placed in the vertical magnetic field of a 6 inch electromagnet mounted on a movable stand. The ultra-high vacuum system was sealed on one end with a bakeable uhv valve, and on the $x$-ray exit port with a 10 mil thick Be window; it was pumped to a base pressure near $10^{-10}$ Torr by a $220 \mathrm{l} / \mathrm{s}$ triode ion pump and a titanium sublimator. The system was mounted $20 \mathrm{~m}$ from the synchrotron ring in a shielded hutch, and isolated from the ring vacuum with a second Be window. A $2 \mathrm{~mm}$ high by $0.75 \mathrm{mrad}$ fan of "white" bending magnet radiation, sharply attenuated below $3 \mathrm{keV}$ by the first $\mathrm{Be}$ window, was 
directed through aperatures in the ring electrode of the trap. Photoions were consequently produced near the minimum of the axial well of the trap, and throughout the radial plane. The photons exiting the system through the second $B$ e window were monitored using an ionization chamber.

The additional mean axial energy gained by the ions through being produced in the electrostatic potential of the trap can be expressed as $E=q D z^{2} / 2 z_{0}^{2}$, where $D$ is the well depth (in $V$ ), $2 \xi$ is the axial extent of the photon beam, and $2 z_{0}=1.6 \mathrm{~cm}$ is the separation of the end electrodes of the ion trap. Since the ions were radially confined by the axial magnetic field $B$, the radial electrostatic field did not contribute significantly to ion kinetic energy, but instead produced a slow drift of the ion cloud about the trap symmetry axis. It was found that the ions could be effectively confined with $D$ as low as $250 \mathrm{mV}$; under these conditions the mean ion energy was near $10 \mathrm{meV}$, corresponding to about $500 \mathrm{~K}$, for all charge states.

The ions were detected by ramping their axial oscillation frequency $\omega_{x}=\left(2 q D / m z_{0}^{2}\right)^{1 / 2}$ through resonance with an excited tuned circuit, composed of a toroidal inductor and the trap capacitance. At resonance, near $140 \mathrm{kHz}$, the ions absorbed $f f$ energy, producing an amplitude modulation proportional to the ion number in each charge state on the of carrier. After amplification and detection, this information was accumulated using a multichannel scalar. The linear sweep was executed from low to high well depths, if storage at the lowest energies was being investigated, or in the opposite direction, if storage at higher wells was desired. Figure 1 shows a sweep starting at a well depth near $5 \mathrm{~V}$, with ions of lowest charge being detected first. The argon ion peaks are quite sharp compared to similar peaks generated by electron impact. This is due to the low energy of the ions, and their narrow distribution in the axial well. Traces of broader peaks, associated with higher energy ions produced via molecular photoionization followed by dissnciation, were sometimes observed in the sweeps starting at deeper wells, if local outgassing had occurred due to the synchrotron radiation beam. 
The stored multi-charged ions can be considered to be a dilute form of a multispecies pure ion plasma (5). Estimates of ion density were possible, based on a dyramical equilibrium between production by synchrotron radiation photoionization, and loss by electron capture collisions (6). Ion loss time constants were measured by blocking the photon beam, using a mechanical shutter rotated by a stepping motor, and observing the decrease of stored ion number with time. The rate of ion production was calculated from the photon flux and K-shell photoionization cross sections (6). For $\mathrm{Ar}^{4+}$, the estimated density was approximately $6 \times 10^{3} / \mathrm{cm}^{3}$, resulting in a Spitzer self-collision time constant (7) $t_{c} \cong 50 \mathrm{~ms}$, and a time constant for thermal equilibration between different charge states $t_{\text {eq }}$ near $100 \mathrm{~ms}$. Thus the ion plasma should relax to thermal equilibrium within typical storage times, and rotate as a rigid body (5). This can only occur if the different charge states move radially to new positions. Since storage times at low pressures can be seconds or more, this situation should prevail at sufficiently high ion densities.

\section{Discussion}

Based on these initial measurements of stored, cold ions, several lines of investigation can be considered. These inciude studies of the plasma properties of the stored ion cloud, measurements of ultra-low energy collision rates of the multi-charged ions with atoms, sequential photoionizaiton to higher stored charge states, and spectroscopic studies of the ions.

\section{a. Plasme Effects}

The radial separation of ion charge states $q_{i}$ and $q_{j}$ depends on the relative size of scale lengths, e.g. $\left(h_{i j}(r)\right)^{-1}=d / d r\left(\left(g_{j}-g_{i}\right) / g_{j}\right)\left(m \omega^{2} r^{2} / 2 k T\right)$. Here $\omega$ is the plasma rotation frequency, $k$ is Boltzmann's constant, and $T$ is the plasma temperature. The scale lengths $\ell_{i j}$ must be $<r_{p}$, the trap radius, for good charge separation, and also much smaller than the Debye length $\lambda_{D}(\mathrm{~cm})=0.74[T(\mathrm{eV})]^{1 / 2}\left[10^{6} / \rho\left(\mathrm{cm}^{-3}\right)\right]^{1 / 2}$, which was $\approx r_{0} / 2$ for

the measured ion parameters. However, the photon flux through the trap can be increased 
in several ways. Using bending magnet radiation, a grazing incidence $x$-ray mirrer is to be installed midway between the source and trap, which is expected to increase the flux through the trap by about a factor of ten. Operation of the NSLS x-ray ring with 350 $m A$ of circulating electrons would increase the useful flux by another factor of five. These improvements alone would reduce the Debye length to about $2.5 \mathrm{~mm}$. There are reports (8) that an electron beam, directed along the trap axis, produces radial cooling of the ion cloud. Such cooling could be easily parformed, since an electron source is already mounted on the trap for test purposes. Radial cooling would draw ions up the radial potential hill, increasing densities near the trap cneter. These modifications would still leave the Debye length comparable to the axial extent of the charge cloud, so even if the scale lengths are appropriately small, the theory may apply only in approximation.

By varying the strength of the confining magnetic field, it may be possible to study the radial distribution of the charges. When ions have a wider distribution of energies, they are lost radially from the trap over a larger range of field $\Delta B$, as $B$ approaches the critical field for containment $B_{c}$ (9). Related effects may be observed for ions with different radial distributions, by studying the ion storage properties as a funciton of the trapping parameters. Alternatively, direct detection of the radial motion may be used.

\section{b. Collision measurements}

Measuring the time constants $\tau_{j}$ for exponential loss of ions by electron transfer collisions in a target gas with density $n$ enables calculation of the rate coefficients $k_{j}=\left(n \tau_{j}\right)^{-1}$ at temperatures near $500 \mathrm{~K}$. Since the mean ion energy is readily estimated, cross sections can be calculated. An interesting case in point is $\mathrm{Ar}^{2+}$ in $\mathrm{Ar}$. Different rates are measured for the ground state and for the metastable states of $\mathrm{Ar}^{2+}$ in flow-drift tube studies at $300 \mathrm{~K}(10)$, but all rates are smaller than $6 \times 10^{-12} \mathrm{~cm}^{3} / \mathrm{s}$. An ion trapping measurement (11) on the ground state at $1.3 \mathrm{eV}$ yielded $k=8.9 \times 10^{-10} \mathrm{~cm}^{3} / \mathrm{s}$. These very different results may mean that $k$ is a rapidly varying function of ion temperature. By measuring the storage time constant of these ions at different well depths, and hence different mean 
energies, data for $k$ at intermediate ion energies could be obtained to test this hypothesis.

\section{c. Sequential Photoionization}

The higher expected photon fluxes using bending magnet radiation discussed above should produce $A r^{4+}$ densities of at least $3 \times 10^{5} / \mathrm{cm}^{3}$, and higher densities of $A r^{2+}$ (sce Fig. 1). Vacancy cascades assuciated with K-shell photoionization of the stored ions should produce a detectable distribution of higher charge states peaking near $\mathrm{Ar}^{7+}$ or $A r^{3+}$. Single electron removal from stored ions by $u v$ synchrotron radiation is discussed in another paper (12). White radiation is useful for sequential photoionization studies since, although the total cross section for photoionization changes little as the outer electrons are removed, the $K$ edge can shift appreciably, e.g. from about $3.2 \mathrm{keV}$ for $\mathrm{Ar}$ to about $4.1 \mathrm{keV}$ for $A r^{16+}$ (13). Wiggler radiation maintains the broadband character of bending magnet radiation, and increases both the photon flux and peak energy, making it a superior choice, particularly if a focusing mirror is available.

Ion charge states can be selectively stored by eliminating other ions using resonant excitation during the production interval. It thus may be feasible to store only two charge states, such as the parent charge $A r^{4+}$, and $A r^{2+}$ which would be formed by photoionizing $A r^{4+}$. Repetition of this process, at low residual pressures, could lead to quite high charge states for study at low energies.

\section{d. Laser Spectroscopy}

Although most transitions in highly-charged ions occur at $\mathrm{X}$-ray energies, a few transitions still fall in the laser range. Several transitions between the fine structure levels of the ground states of $A r^{0+}, A r^{10+}$, and $A r^{12+}$ occur at wavelengths between $4500 \mathrm{~A}$ and $6800 \lambda(14)$, easily accessible to $c w$ or puloed dye lasers. Since $v / c$ for the ions is near $1.5 \times 10^{-6}$, Doppler widths should be less than $1 G H z$. The possibilities inherent in these considerations for laser cooling (15) highly charged ions to still lower mean energies in the trap is particularly interesting, although the relatively slow decay rates of the levels may inhibit the effectiveness of this method. 


\section{Conclusion}

Measurements on multi-charged ions produced using synchrotron $x$-ray radiation in a Penning ion trap have been described. Several research topics using these ions have been suggested. The low ion energies inherent in this method should make feasible research which is difficult to accomplish by other means.

\section{Acknowledgments}

This research was supported by the National Science Foundation, and by the Department of Energy under Contract DE-AC02-76CH00016.

\section{References}

1. Carlson, T. A., Hunt, W. E., and Krause, M. O., Phys. Rev. 151, 41 (1966).

2. Short, R. T., O, C. S., Levin, J. C., Sellin, I. A., Liljeby, L., Huldt, S., Johansson, S.-E., Nilsson, E., and Church, D. A., Phys. Rev. Letters 56, 2614 (1986).

3. Church, D. A., Jones, K. W., Johnson, B. M., Meron, M., and Sellin, I. A., J. Phys. B17, L401 (1984).

4. Johnson, B. M., Meron, M., Agagu, A., and Jones, K. W., Nuc. Instrum. Meth. B24/25, 391 (1987).

5. O'Neil, T. M., Phys. Fluids 24, 1447 (1981).

6. Church, D. A., Kravis, S. D., Sellin I. A., O, C. S., Levin, J. C., Short, R. T., Meron, M., Johnson, B. M. and Jones, K. W., Phys. Rev. A (in press).

7. Spitzer, Jr., L., "Physics of Fully Ionized Gases", (Wiley, New York, 1956).

8. Van Dyck, Jr., R. S., and Moore, F. L., communication at the Workshop and Symposium on the Physics of Stored and Trapped Particles, Stockholm (1987).

9. Holzscheiter, H. M., Nuc. Instrum. Meth. A240, 457 (1985).

10. Johnson, R., and Biondi, M. A., Phys. Rev. A18, 996 (1978).

11. Prior, M. H., Phys. Rev. A30, 3051 (1984).

12. Meron, M., Johnson, B. M., Jones, K. W., and Church, D. A., these proceedings.

13. Reilman R. F., and Manson, S. T., Astrophys. J. Supp. 40, 815 (1979).

14. Bashkin, S., and Stoner, Jr., J. O., "Atomic Energy Levels and Grotrian Diagrams, Vol. 2" (North-Holland Amsterdam (1978)).

15. Hänsch, T. W. and Schawlow, A. L., Opt. Commun. 13,68 (1975); Wineland, D., and Dehmelt, H. G., Bull. Am. Phys. Soc. 20, 637 (1975). 


\section{Figure Caption}

Figure 1. Argon ion $q / m$ signals obtained from ions stored in the trap near $5 \mathrm{~V}$ well by molecular photoionization and dioad background sigmals due to hot ions produced from +2 through +6 (left to right). 


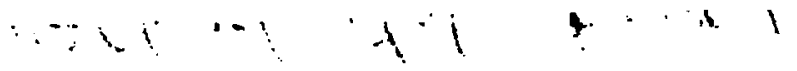

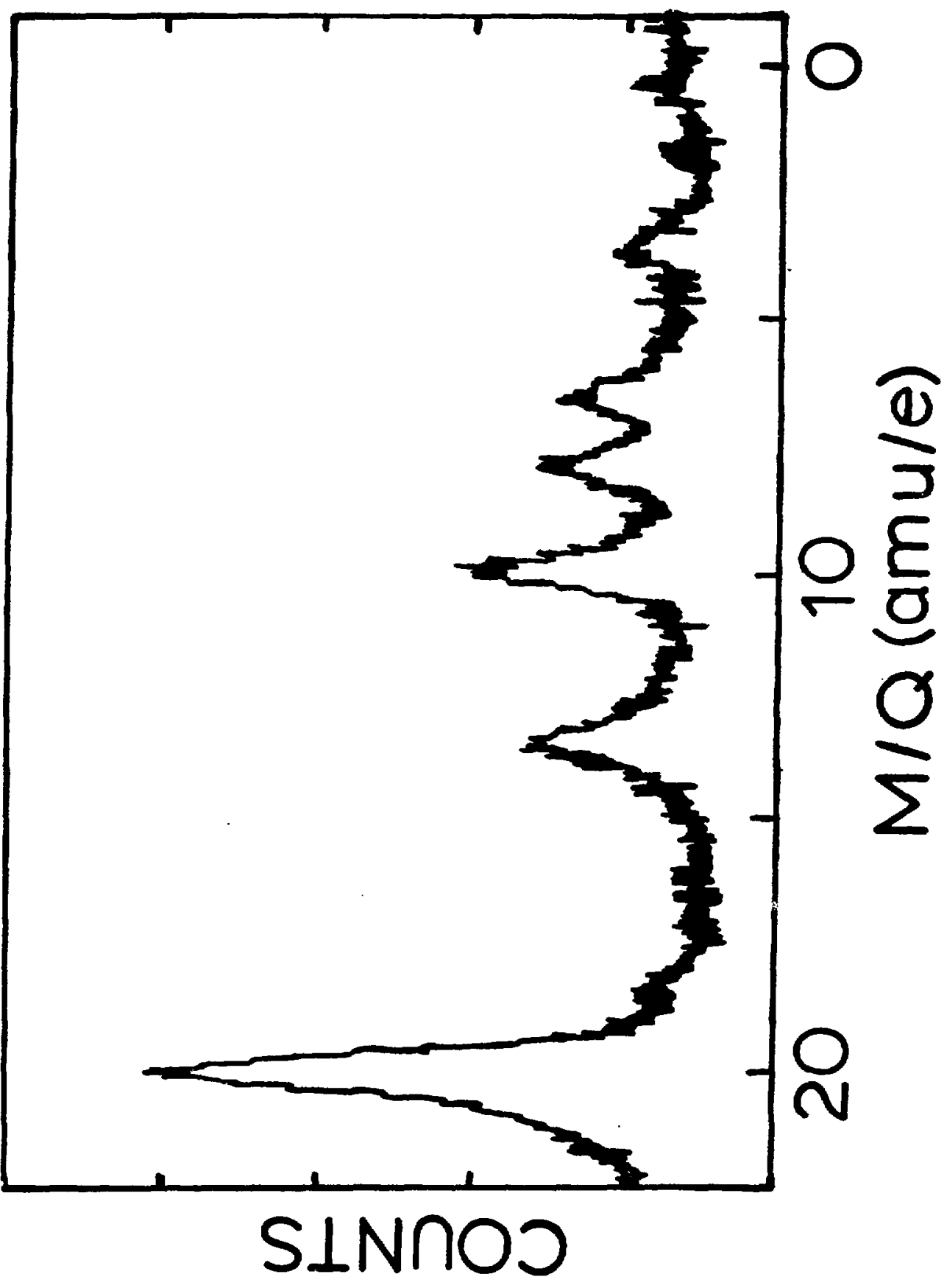




\section{DISCLAIMER}

This report was prepared as an account of work sponsored by an agency of the United States Government. Neither the United States Government nor any agency thereof, nor any of their employees, makes any warranty, express or implied, or asumes any legal liability or responsibility for the accuracy, completeness, or usefulness of any information, apparatus, product, or process disclosed, or represents that its use would not infringe privately owned rights. Reference herein to any specific commercial product, proces, or service by trade name, trademark, manufacturer, or otherwise does not necesuarily constitute or imply its endorsement, recomendation, or favoring by the United States Government or any agency thereof. The view and opinions of authors expressed berein do not necesarily state or reflect those of the United States Government or any azency thereof. 